\title{
Rail weld geometry and assessment concepts
}

M J M M Steenbergen* and C Esveld

Railway Engineering, Faculty of Civil Engineering and Geosciences, Delft University of Technology, Delft, The Netherlands

The manuscript was received on 15 September 2005 and was accepted after revision for publication on 22 February 2006.

DOI: $10.1243 / 09544097 J R R T 38$

\begin{abstract}
Worldwide, metallurgical rail welds are being geometrically assessed by the principle of vertical deviations satisfying given tolerances, measured with steel straightedges or occasionally with digital/electronic straightedges. In this approach, the geometrical shape of the weld in longitudinal direction has no real influence, although it has a direct relation with the dynamic wheel-rail interaction forces, which are responsible for track deterioration. In this article, different new assessment methods for rail welds are proposed and evaluated in practice, after which a choice is made for the best method. This is done in line with the situation in the Netherlands, where the chosen new method was recently introduced and standardized (2005). The proposed method is based on a limitation of the gradient of the discrete measurement signal, implying a limitation of the wheel-rail dynamic contact force.
\end{abstract}

Keywords: rail welds, welding, weld geometry, rail joints, weld assessment

\section{INTRODUCTION}

The step from railway tracks with bolted rail joints to tracks with continuously welded rails (CWRs) was an important improvement of the geometry of the interface between wheel and rail. However, rail weld surface irregularities (especially in situ made thermit and flash butt welds) in CWR tracks remain a source of high-frequency excitations of both the track and the vehicle, as the quality of the vertical geometry along the rail is always less than that of new, straightened rail or ground rail. Especially, on high-speed lines, the continuity of the rail surface in longitudinal direction is of great importance.

With respect to the track, high-frequency excitations are most detrimental for concrete sleepers, rail fastenings, and other track components and responsible for non-uniform ballast settlement or local decompaction. Concerning the vehicle, highfrequency excitations may introduce non-linearities in wheel-rail contact, causing non-continuous

*Corresponding author: Railway Engineering, Faculty of Civil Engineering and Geosciences, Delft University of Technology, Section of Road and Railway Engineering VBK, PO Box 5048, NL 2600 GA Delft, The Netherlands. email: m.j.m.m.steenbergen@tudelft.nl railhead damage and wheel flats, as the wheel acts as a grinding element on both rim and railhead. These high-frequency excitations finally lose their energy by dissipation (plastic deformation, irreversible creep, and other processes related to the growth of track damage) and migration to lower frequency vibrations of other train and track components. From the above point of view, it is crucial to realize a vertical weld geometry as close as possible to a straight line. This asks for appropriate assessment concepts. In this article, different new assessment methods for rail welds are discussed. One of them is proposed for practice and evaluated. This is done in line with the situation in the Netherlands, where this new method has been recently standardized (2005) [1, 2].

\section{PRACTICAL REQUIREMENTS ON ASSESSMENT METHODS}

The geometrical shape of the rail weld has a direct relation with the magnitude of the dynamic wheelrail interaction force. Both the frequencies and the corresponding magnitudes of this force are related with the growth rate of damage and deterioration of the different track components. 
It is presupposed that for geometrical assessment, a digital, electronic straightedge is used to measure the weld, so that the geometrical data are available after measurement. Then, an optimal approach to weld assessment would be the evaluation of the spectrum of the contact forces occurring for the weld under consideration, where the spectral amplitudes should not exceed predefined values. This would require the computation of the dynamic contact force between wheel and rail weld, followed by a spectral analysis in the frequency domain. If the considered weld does not satisfy the requirements, then the weld geometry should subsequently be changed in such a way that it would satisfy the norms. As these norms are expressed in terms of forces and not of geometry, an additional computational effort is required to determine the most efficient way to arrive at an acceptable geometry in an iterative process.

A simplified approach would be to calculate the dynamic contact force in the time domain for the considered weld, where the maximum force should not exceed a predefined value.

These methods have two disadvantages, which make them unsuitable in practice. First, the magnitude of wheel-rail contact forces depends on the properties and the configuration of the track structure to a large extent. Therefore, the influence of the local track properties should be accounted for, which makes a uniform assessment, independent of the track type and component properties, impossible. A second problem is of a computational nature. Basically, the solution of the dynamic wheel-rail contact problem in a linear form asks for the solution of a set of differential equations (of second order). It is not feasible in practice to perform these calculations for each separate rail weld each time it is being measured.

However, in general, it may be stated that the 'smoothness' of the geometry is a measure for the occurring dynamic forces between wheel and rail, and therefore, for the rate of track deterioration. This concept will be elaborated in the following sections. The main objective is to develop a practical tool for the assessment of the geometry of rail welds, by directly relating the vertical rail geometry to the magnitude of dynamic contact forces which occur for that geometry, without having to perform complex dynamic calculations for each separate geometrical measurement.

\section{WHEEL-RAIL DYNAMICS FOR SHORT LENGTH-SCALE DISTURBANCES IN THE INTERFACE GEOMETRY: MODEL SIMPLIFICATIONS}

The velocity for trains generally ranges between 40 and $300 \mathrm{~km} / \mathrm{h}$, with operational speeds for conventional passenger trains of $\sim 140 \mathrm{~km} / \mathrm{h}$ and for freight trains of $\sim 80-100 \mathrm{~km} / \mathrm{h}$. This yields a most relevant velocity range of $\sim 20-40 \mathrm{~m} / \mathrm{s}$. Given relevant wavelengths in a range of $0.1-2 \mathrm{~m}$ (as will be discussed in section 4), the corresponding most relevant frequency range of excitation of the wheel-rail system can be found within $10-400 \mathrm{~Hz}$ (which is the low- and mid-frequency range).

In the train-track dynamics, several stiffness factors and masses play a role in this frequency range for the determination of the wheel-rail contact forces. The most important masses to be mentioned are the unsprung mass $M_{\text {unsprung }}$ and the equivalent track mass $M_{\text {track }}$; the most important stiffnesses are the primary suspension stiffness $k_{1}$, the wheel-rail Hertzian contact stiffness $k_{\mathrm{H}}$, and the equivalent track stiffness $k_{\text {track }}$. Damping is not of primary importance at the wheel-rail interface. In a most general form, the dynamic wheel-rail contact force as a function of time may be written as

$$
F_{\text {dyn }}(t)=f\left(z(x), V, M_{\text {unsprung }}, M_{\text {track }}, k_{1}, k_{\text {Hertz }}, k_{\text {track }}\right)
$$

where $z(x)$ is the vertical rail geometry in the longitudinal direction and $V$ is the train speed.

For each frequency in the frequency domain, a certain mass in combination with a related stiffness will have a dominating role in absorbing the excitation energy and determine the magnitude of wheel-rail contact forces. For the band with the lowest frequencies, the combination of wheel mass on track stiffness will be dominating; for increasing frequency, the role of the wheel mass as the dominating mass will be taken over by the equivalent track mass; and for the highest part of the frequency range, the Hertzian stiffness will replace the track stiffness.

Now, a quasi-static response of the dominating mass-stiffness combination is assumed. This will allow for a reduction of the dynamic contact problem, in terms of second-order differential equations, to an algebraic problem, as has been discussed in section 2. If the minimum relevant wavelength $L$ in the rail irregularity is taken as $0.1 \mathrm{~m}$ (section 4), the excitation frequency at a train velocity $V(\mathrm{~m} / \mathrm{s})$ equals $f=V / L=10 V(\mathrm{~Hz})$, from which the requirement for $f$ can be derived directly. A quasi-static response occurs for $f<0.5 f_{0}$ approximately, where $f_{0}$ is the natural frequency of the mass-spring system under consideration, to be determined from the dominant mass $M$ and stiffness $K$. The resulting requirement for quasistatic response is given by (using SI units)

$$
V<\frac{1}{40 \pi} \sqrt{\frac{K}{M}}
$$




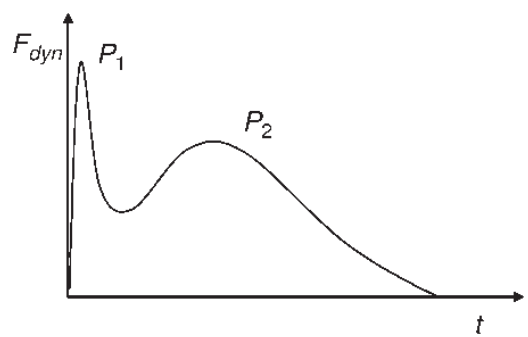

Fig. 1 Qualitative behaviour of $P_{1}$ and $P_{2}$ dynamic wheel-rail interaction forces at an interface irregularity on ballasted track [5]

To which extent this requirement is satisfied depends on the specific track properties and must be verified by measurements. The train velocity $V$ herein is given by the line-section speed in question.

In general, the unsprung axle mass is $\sim 1800 \mathrm{~kg}$ for conventional trains [3]. In general, near weld irregularities in ballasted track, the ballast is not wellcompacted below the sleepers (the problem of 'fluttering' sleepers with voids underneath arises after some track use). Therefore, the track stiffness may be assumed - for small vibration amplitudes to be determined by the rail bending stiffness, where the rail deflection is largely quasi-static and the contribution of high-frequent modes can be neglected. Assuming now an equivalent track mass consisting of two fully effective concrete sleepers of each $300 \mathrm{~kg}$ [4] and $4 \mathrm{~m}$ of effective rail mass (60 kg/m; UIC 60), the equivalent track mass can be estimated at $850 \mathrm{~kg}$. This is $<50$ per cent of the unsprung axle mass. For this reason, the wheel mass is considered as fixed in the vertical direction during the time interval that the wheel passes the weld irregularity.

In this context, also the traditional concept of $P_{1}$ and $P_{2}$ forces, which was originally developed in a somewhat different context, for insulated or bolted rail joints [5], can be used (Fig. 1). Owing to the difference in inertia between unsprung wheel mass and equivalent track mass, the response of the wheel to the relatively short-length excitation will show a certain delay relative to the response of the track (rail and sleepers). The $P_{1}$ force, which is a quasi-instantaneous amplification of the wheelrail contact force, originates from the reaction of the track and is mainly determined by the track equivalent stiffness and the rail equivalent mass. This reaction may be quasi-static for irregularities with a somewhat longer length scale and/or relatively low velocities or dynamic for shorter length scales and/or higher velocities. In this context, 'quasi-static' refers to the system governed by the Hertz contact stiffness and the track equivalent stiffness in parallel and the equivalent track mass (Fig. 2(a)). The time scale of the peak is determined by the length scale of the irregularity and the actual train speed or, alternatively, by the frequency of the governing mass-stiffness combination.

The $P_{2}$ force results from the reaction of the axle box to the excitation. Its magnitude is determined mainly by the unsprung wheel mass and the equivalent track stiffness, and the time scale of the peak is larger because of the lower eigenfrequency of the dominating mass-spring system when compared with the system consisting of equivalent track mass and stiffness. Owing to the $P_{2}$ force, the sleepers directly after the weld are often observed to be well fixed in the ballast bed, as they are pressed into the ballast by all passing axles. $P_{1}$ and $P_{2}$ may be considered to be proportional, i.e. the magnitude of $P_{2}$ is determined by $P_{1}$.

Figure 2(b) shows the dominating mass-stiffness combination resulting from the earlier assumptions, disturbed by the weld irregularity $z(x)$ moving at train speed $V$ and valid during the time interval of passage of the weld. As the Hertz contact is far more stiff than the equivalent track stiffness, it is assumed to be rigid in the model.
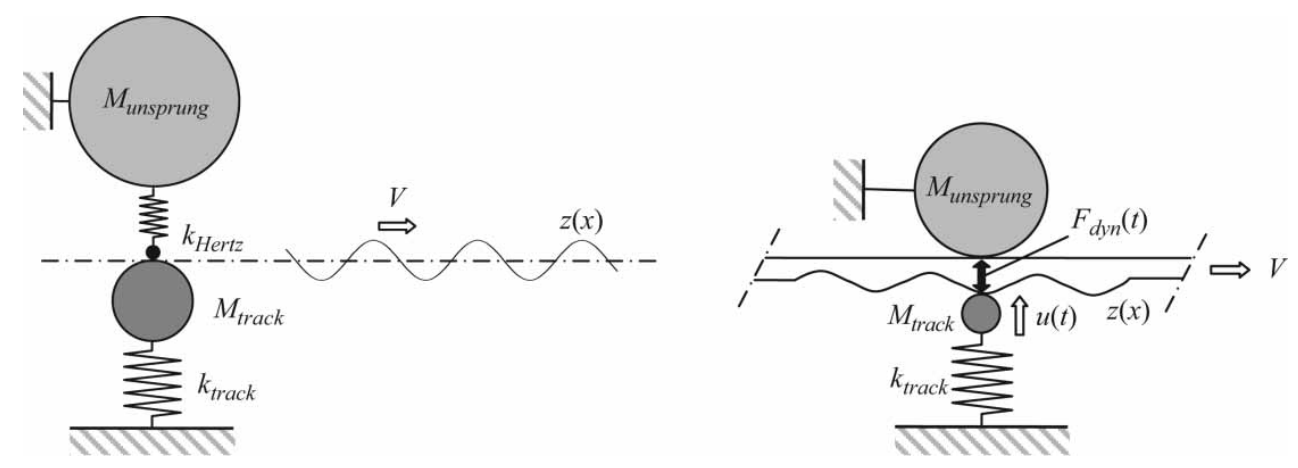

Fig. 2 Mass-stiffness combinations in the wheel-rail system (a) and disturbance by a rail irregularity (b) 
The equation of motion of the system in Fig. 2(b) is given by

$$
M_{\text {track }} \ddot{u}(t)+k_{\text {track }} u(t)=0
$$

A quasi-static response of the equivalent track mass (in the system depicted in Fig. 2(a)) was assumed, yielding

$$
u(t)=z(t)
$$

The dynamic contact force in the model of Fig. 2(b)) is then given by

$$
F_{\text {dyn }}(t)=k_{\text {track }} u(t)=k_{\text {track }} z(t)
$$

and it is directly proportional to the excitation as a function of time. Using equation (4), this expression may be written alternatively in terms of the second derivative of the excitation as a function of time

$$
F_{\text {dyn }} \sim M_{\text {track }} \ddot{z}(t)
$$

Thus, the dynamic component of the $P_{1}$ contact force between wheel and track is approximated as the inertia force originating from the track mass, which follows the vertical irregularity. The introduction of a dimensionless calibration factor $\beta$ yields

$$
F_{\text {dyn }}=\beta M_{\text {track }} \ddot{z}(t)
$$

where $\beta$ accounts for dynamic influences which were not modelled, inaccuracies introduced by assumptions on the response and non-linearities; its value can be chosen as a discontinuous function of the line-section speed and should be determined from the validation measurements. Using the equality $\mathrm{d}^{2} z / \mathrm{d} t^{2}=V^{2}\left(\mathrm{~d}^{2} z / \mathrm{d} x^{2}\right)$, this expression for the dynamic contact force can be transformed to the space domain

$$
F_{\mathrm{dyn}}=\beta M_{\mathrm{track}} V^{2} \frac{\mathrm{d}^{2} z}{\mathrm{~d} x^{2}}
$$

The maximum of this expression, for a certain velocity, is found for the maximum of the spatial second derivative of the disturbance $z(x)$

$$
F_{\text {dyn, max }}=\beta M_{\text {track }} V^{2}\left|\frac{\mathrm{d}^{2} z}{\mathrm{~d} x^{2}}\right|_{\text {max }}
$$

Using a quasi-static approach, the second derivative of the geometry thus becomes a direct measure for dynamic contact forces.

In Fig. 3, finite-element simulation results (obtained with the DARTS-NL software [6]) are shown for a train vehicle, passing an artificial weld irregularity in the track. The irregularity is a smooth 'wave' with an amplitude of $1 \mathrm{~mm}$ and a length of $1 \mathrm{~m}$. The train speed $V$ equals $140 \mathrm{~km} / \mathrm{h}$. The following parameter values have been adopted in the

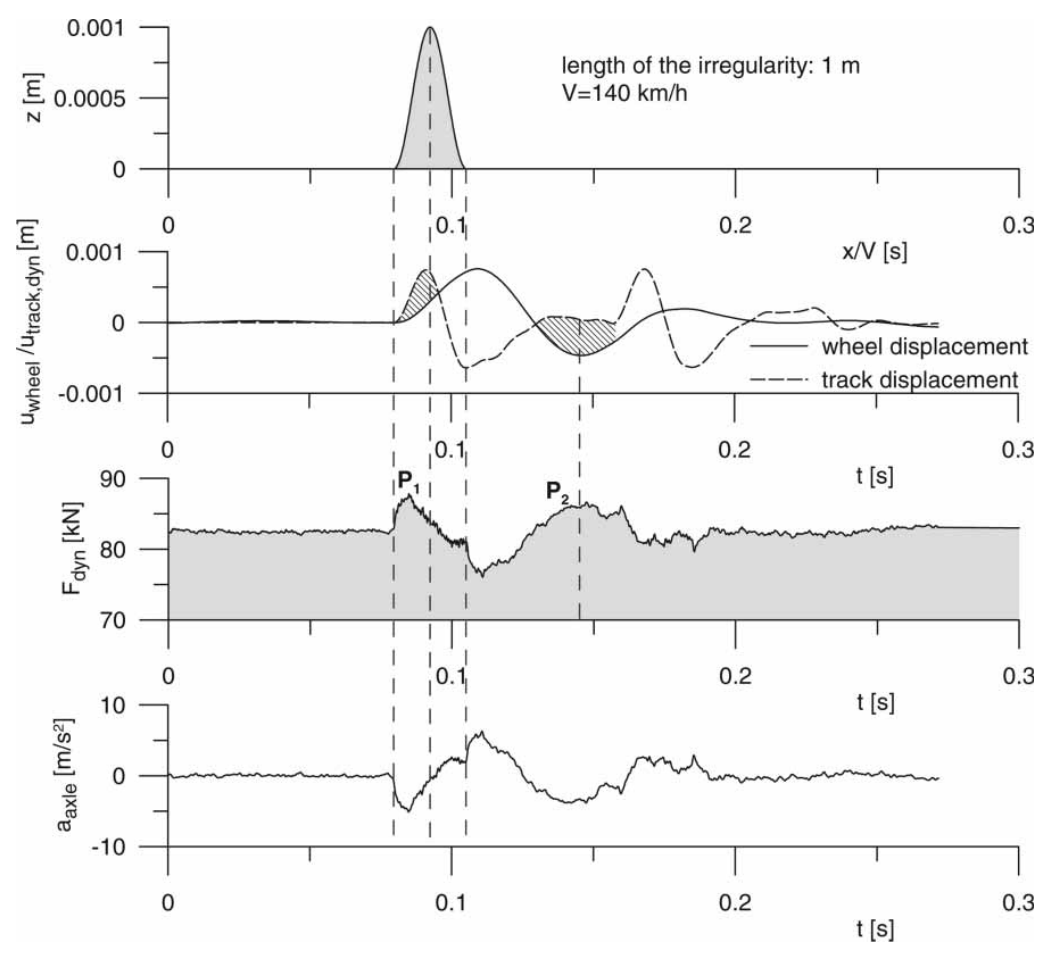

Fig. 3 Response of the wheel-rail system to a short-length irregularity 
calculation: a wheel mass (half unsprung mass) of $970 \mathrm{~kg}$; a sleeper mass of $300 \mathrm{~kg}$; UIC 54 rail; a primary suspension stiffness of $1.8 \cdot 10^{6} \mathrm{~N} / \mathrm{m}$ (per wheel); a rail pad stiffness of $1.2 \cdot 10^{9} \mathrm{~N} / \mathrm{m}$, and a ballast stiffness of $30 \cdot 10^{6} \mathrm{~N} / \mathrm{m}$ per sleeper, where the latter value is rather small to account for the generally bad compaction of the ballast underneath the sleepers close to the irregularity. A non-linear Hertz contact model has been used. The following quantities are shown in Fig. 3, where all parameters are functions of time.

1. The geometry of the irregularity $z$.

2. The vertical wheel displacement $u_{\text {wheel }}$ of the first wheel of a passing bogie, relative to the displacement resulting from the quasi-static vertical track deflection because of the passage of the static axle load; the displacement is defined positive in upward direction and calculated in a moving coordinate frame.

3. The dynamic displacement $u_{\text {track,dyn }}$ of the rail/ track relative to the quasi-static deflection calculated at the centre of the irregularity. This displacement is defined positive in downward direction and calculated in a fixed coordinate system.

4. The wheel-rail contact force $F_{\text {dyn }}$ for the concerned wheel (the dynamic force is superimposed on the static value) in a moving coordinate frame.

5. The axle box acceleration $a_{\text {axle }}$ in a moving coordinate frame.

The repetitive pattern in the track displacement in Fig. 3 is due to the passage of the second axle of the train bogie. From Fig. 3, it can be observed that the assumptions that have been made in this paragraph correspond well to the real system response. The track mass directly follows the irregularity in an almost quasi-static manner, whereas the response of the axle mass shows a delay relative to the irregularity. It can be shown that this effect is even stronger for a non-smooth irregularity with a shorter length scale. The $P_{1}$ force, resulting from the instantaneous reaction of the track to the irregularity, as well as the $P_{2}$ force, resulting from the reaction of the unsprung mass, can be clearly distinguished. The magnitude of both peaks follows from the hatched area in Fig. 3. Finally, it is observed that the dynamic contact force is proportional to the axle box acceleration.

\section{PRELIMINARY OPERATIONS ON MEASUREMENT DATA}

Usually, the longitudinal rail weld geometry is measured with a straightedge with $1 \mathrm{~m}$ basis [7].
Other (mostly longer) bases exist, but $1 \mathrm{~m}$ is a uniform choice. Further, it is measured at the centre of the top of the railhead in the cross-sectional plane. For connections between worn and new rails, there are other possibilities, as a worn railhead is no longer symmetric; however, because a uniform choice has to be made for standardization, commonly the centre of the railhead is taken.

When a digital straightedge is used, the sampling of the rail geometry is a discrete process. Therefore, a minimum wavelength exists that can be registered for rail welds. This minimum wavelength is described by a minimum of five sampled coordinates or four sampling intervals (Fig. 4).

Measurement devices sample the vertical rail geometry with an interval of $5 \mathrm{~mm}$. Before starting operations on the measured signal, it is averaged over a distance of $25 \mathrm{~mm}$ (including five data points); the resulting data points have an interval of $25 \mathrm{~mm}$. The reason of this averaging process is to avoid very noisy signals (especially for the derivatives) because of irregularities on microscale. These irregularities, with length scale in the same order of magnitude as the width of the contact patch on the railhead, are physically not relevant because of the local plastic railhead deformation and wear after some track use. An example of this filtering process is given in Fig. 5.

Using a $25 \mathrm{~mm}$ interval, as explained earlier, leads to a minimum full wavelength for welding irregularities of $0.1 \mathrm{~m}$. This corresponds well to the situation in practice. Smaller wavelengths may exist in theory, but in reality, these components have such small amplitudes (Fig. 5) that they will have disappeared from the signal by both wear and plastic deformation after some wheel passages.

In this way, a global range of $0.1-2 \mathrm{~m}$ for the relevant wavelengths in the weld irregularity can be established. The largest wavelength of $2 \mathrm{~m}$ in this range corresponds to geometrical deviations of the end parts of rail sections (resulting from straightening by the rollers in the production process), which are welded together, or the 'set-up' of both rail ends by the welding crew or the welding machine before welding them together.

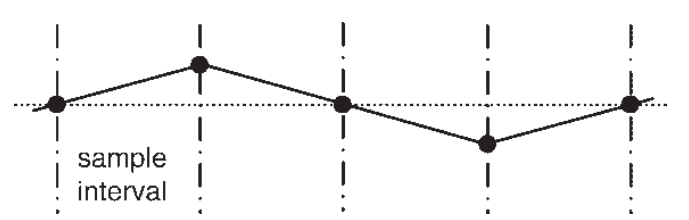

Fig. 4 Minimum number of sampling points to describe a single wavelength 


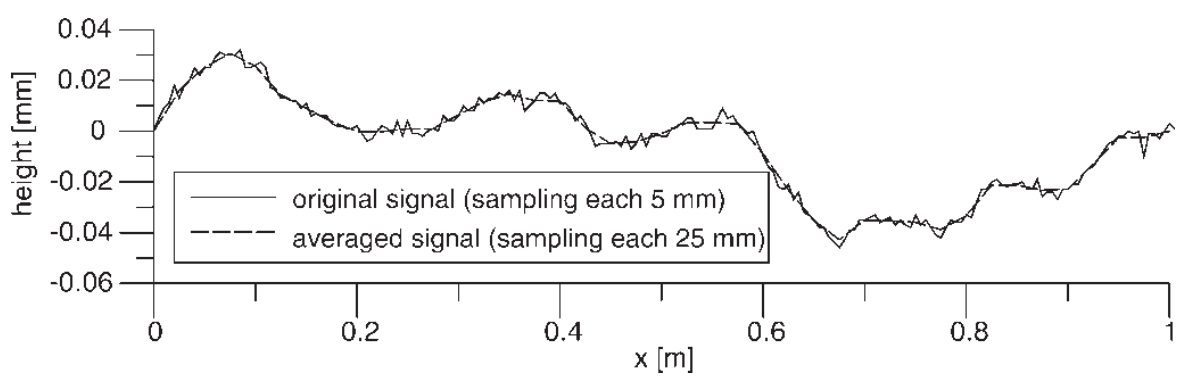

Fig. 5 Measured longitudinal rail geometry and averaged signal

\section{GEOMETRICAL ASSESSMENT METHODS, COMPARISON, AND ASSESSMENT PROPOSAL FOR CONVENTIONAL TRACKS}

The 'smoothness' of the sampled geometry of a weld can be quantified in a number of ways. To investigate which parameter is most suitable to describe and assess its quality, the following methods are distinguished:

(a) zeroth derivative-based method, based on a limitation of the sampled original geometry;

(b) first derivative-based method, based on a limitation of the first derivative or gradient of the sampled geometry;

(c) second derivative-based method, based on a limitation of the second derivative of the sampled geometry.

Method (a) is the usual approach, where vertical tolerances have to satisfy certain requirements. These requirements are usually independent of the line-section train speed. It is clear that the contact force corresponding to the weld geometry is an increasing function of $V$. This means that for a specific weld geometry satisfying the requirements, in a part of the network designed for $140 \mathrm{~km} / \mathrm{h}$, the allowable contact forces are much higher than for the same weld in a marshalling yard, which is inconsistent.

A typical example of the result of the current rail welding methods (especially for in situ welding) and their assessment are shown in Fig. 6. Both rail ends are 'set-up' under a small angle in vertical direction (usually the overlift is $1-2 \mathrm{~mm}$ on a

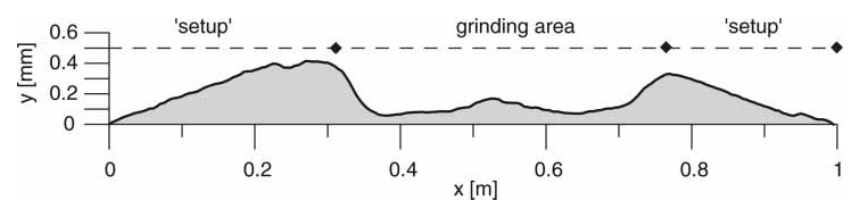

Fig. 6 Typical geometry example of an in situ made rail weld, resulting from current methods and standards
1-1.2 $\mathrm{m}$ basis [7]). After welding, the resulting 'roof', which does not fall within the maximum tolerance, is ground off. The result is often far from smooth and introduces large dynamic amplifications in contact forces.

Assessment method (a) can only be enhanced by differentiating on the line-section speed. Therefore, this method is not discussed any further, and in the following, the focus is on methods (b) and (c).

In Fig. 7, an example is given of the application of both methods (b) and (c) for a weld geometry measurement. Similarly as in section 4 , the measurement data are averaged and filtered before calculating the derivatives. The averaged measurement signal and the first and second derivatives are shown, each normalized by the respective maximum of their absolute value. It is clear that both methods provide a good evaluation of the 'smoothness' of the wheel-rail interface geometry.

The maximum dynamic contact force for a weld is a function of the train speed (equation (9)), and therefore, a differentiation on line-section velocity is introduced. The following speed intervals are chosen (according to the standards in The Netherlands [7]): 0-40, 40-80, 80-140, and 140$200 \mathrm{~km} / \mathrm{h}$.

To enable a comparison between methods (b) and (c), the so-called weld quality index (QI) is introduced as a relative measure of the 'dynamic quality' of a certain rail weld geometry. This QI is a

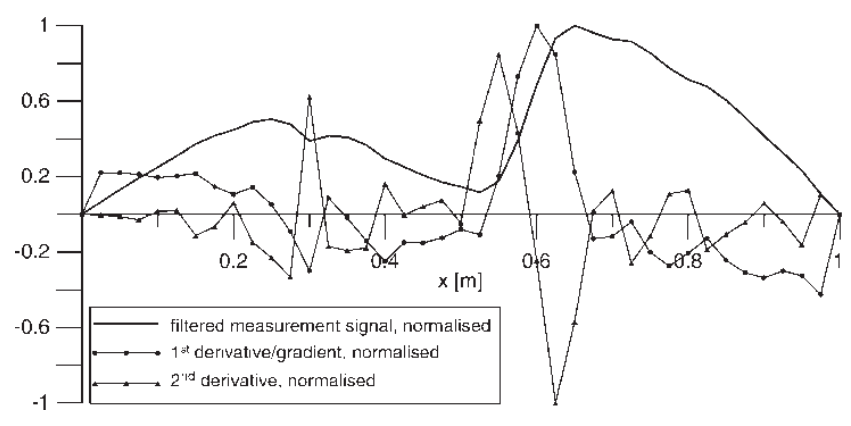

Fig. 7 Averaged discrete measurement signal, first and second derivatives, each normalized with their maximum absolute value 
non-dimensional number, which is defined as the ratio of the calculated maximum of the absolute value of the derivative (first or second) per weld to the corresponding intervention level for the considered train velocity range. Thus, a QI less than or equal to 1 leads to acceptance of the weld and a value larger than 1 leads to rejection.

On the basis of experience from practice, empirical intervention levels have been determined for both approaches (b) and (c), by performing an analysis of a representative weld population (a sample of 72 rail welds) for the different speed regimes [1]. Implementation of the quadratic influence of the train speed appears to be not feasible, as it leads to excessive values; therefore, an influence of the velocity with a power smaller than 2 has been adopted. The adopted intervention levels are given in Table 1, based on which the QI can be determined for a specific weld, for a given line-section speed.

A comparison between both assessment methods (b) and (c) for the considered weld population shows that, in general, approach (b) leads to less extreme values than approach (c). This also follows from the average scores for the analysed population. According to approach (c), average QIs were 1.2, 1.8, 3 , and 3 for the four considered velocity ranges, and $1.2,1.6,2$, and 2.2 according to approach (b), respectively, which is much more moderate. Further, method (c) turns out to be most sensitive to very small, short-length irregularities (with a length scale of some centimetres) and, on the contrary, not very sensitive for longer irregularities (with a global length scale of $0.5 \mathrm{~m}$ ). This is not the case for approach (b).

Very small, short-length irregularities (indentations) often occur after rail welding at both sides of the weld material because of both shrinkage after grinding and further cooling down and differences in steel hardness along the rail surface with local minima in the heat-affected zones at both sides of the weld metal [7]. In Fig. 8(a), an example is given of an almost perfectly straight weld, showing

Table 1 Empirical intervention levels for first and second derivative of the longitudinal rail weld geometry differentiated on line-section speed

\begin{tabular}{|c|c|c|}
\hline $\begin{array}{l}\text { Intervention level } \\
\text { for the discretized } \\
\text { weld geometry Speed } \\
\text { interval }(\mathrm{km} / \mathrm{h})\end{array}$ & $\begin{array}{l}\text { Gradient } \\
\text { (mrad) }\end{array}$ & $\begin{array}{l}\text { Second } \\
\text { derivative } \\
{\left[10^{-3} \mathrm{~m}^{-1}\right]}\end{array}$ \\
\hline $0-40$ & 3.20 & 202.50 \\
\hline $40-80$ & 2.40 & 101.25 \\
\hline $80-140$ & 1.80 & 82.65 \\
\hline $140-200$ & 0.90 & 81.00 \\
\hline
\end{tabular}

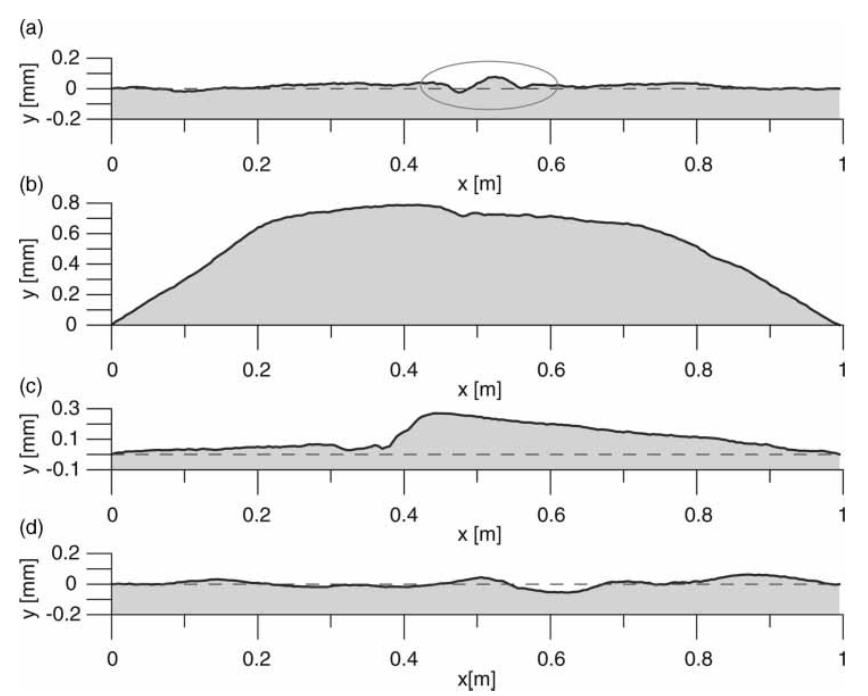

Fig. 8 Measurement examples of longitudinal weld geometries (a) with indentations due to nonuniform shrinkage after welding and grinding; (b) with an irregularity with longer length scale; (c) with a step; and (d) with negative height coordinates

these indentations. The weld in Fig. 8(a) has QIs $1.3,1.7,3.3$, and 3.3 (for the four speed intervals) according to (c) and indices 0.6, 0.8, 1, and 1.1 according to (b). In Fig. 8(b), an example is shown of the geometry of a weld with an irregularity with a longer length scale (the weld is of bad quality, with maximum height of $0.8 \mathrm{~mm}$ ). The weld has QIs 0.6, 0.9, 1.4, and 1.4 according to (c) and 1.1, 1.4, 2, and 2 according to (b). As the assessment concept (c) has these drawbacks, concept (b), based on first derivatives or geometrical gradients, proves to be most appropriate for practical implementation.

In Figs 8(c) and (d), examples are shown of the practical differences between the current assessment method (a) and the proposed method (b). The weld geometry in Fig. 8(c), which shows an aggressive step, is accepted according to many current standards (which allow a vertical tolerance of $+0.3 \mathrm{~mm}$ on a $1 \mathrm{~m}$ basis for many conventional tracks and on a $1.6 \mathrm{~m}$ basis for the French high speed (TGV) track [7]). However, according to the newly proposed method based on the gradient of the signal, the weld has indices 1.1, 1.7, 2, and 2.5 for the four speed intervals and should be rejected. In Fig. 8(d), an example is shown of an almost perfect weld. However, as it has some negative height coordinates, it is rejected according to the current standards, which do not allow negative values. According to the newly proposed method, the weld has indices 0.5, 0.7, 0.9, and 1 and is, therefore, accepted for all speed intervals. 


\section{GEOMETRICAL CORRELATION BETWEEN THE ORIGINAL GEOMETRY AND THE FIRST AND SECOND DERIVATIVES OF A DISCRETE SIGNAL WITH A LIMITED EXTREME GRADIENT}

In the previous, the establishing of intervention levels for the original weld geometry $z(x)$ and its derivatives $\mathrm{d} z / \mathrm{d} x$ and $\mathrm{d}^{2} z / \mathrm{d} x^{2}$ were considered as methods of assessment, where $z(x), \mathrm{d} z / \mathrm{d} x$, and $\mathrm{d}^{2} z / \mathrm{d} x^{2}$ were treated as independent variables (in the sense that standardization of the maximum of one of them does not influence extreme values of the other variables). However, limitation of the extremes of the first or second derivative of a discrete spatial signal with limited length implicitly leads to a limitation of the geometry itself and the remaining derivative. This phenomenon will be the subject of this paragraph.

To determine the relations between the original geometry and the derivatives of the sampled geometry when their extreme values are limited, the following quantities are introduced, along with their definitions:

(a) $\theta$ : the angle of the signal with the horizontal axis or the slope in an arbitrary sample interval; its standardized maximum is denoted as $\alpha$;

(b) $\tan \theta$ : the discrete gradient of the signal in sample interval $i$ according to $\tan \theta_{i}=\left(z_{i+1}-z_{i}\right) / d$; its standardized maximum equals $\tan \alpha$;

(c) $\Delta \theta$ : the relative angle (slope) of the signal in two successive intervals at the sample position;

(d) $\Delta \tan \theta$ : the relative discrete gradient of the signal in two successive intervals or the discrete transition in the gradient at the sample position;

(e) $\mathrm{d} z / \mathrm{d} x$ : the first derivative of the signal at sample position $i$ according to $\mathrm{d} z_{i} / \mathrm{d} x=\left(z_{i+1}-z_{i-1}\right) / 2 d$;

(f) $\mathrm{d}^{2} z / \mathrm{d} x^{2}$ : the second derivative at sample position $i$ according to $\mathrm{d}^{2} z_{i} / \mathrm{d} x^{2}=\left(z_{i+2}-2 z_{i}+z_{i-2}\right) / 4 d^{2}$.
To be able to work with continuous expressions for the contact force in time in a later stage, also the vertical rail geometry must be defined in the interval between the sampling positions. The most basic linear interpolation of the signal is chosen to obtain a continuous signal.

In Figs 9(a) and (b), the above quantities are shown and compared for a sampled harmonic signal with the minimum wavelength $4 d$. Comparison of Figs 8(a) and (b) shows that, for a harmonic signal, the values of the gradient and the first derivative are equal in the sampling positions, which is not true for the relative gradient and the second derivative.

Now, the special case is considered that the discretely sampled rail geometry, obtained after the averaging and filtering process, is a purely harmonic signal with a gradient in each interval at the intervention level (Figs 10 and 12). In this particular case, one wavelength is fully described by five sampling points (the wave is quadri-linear). The sample interval is $d=25 \mathrm{~mm}$ and the signal length is $l=1 \mathrm{~m}=40 d$. The minimum wavelength is given by $L_{\min }=4 d$ ( $=100 \mathrm{~mm}$ ); the maximum equals $L_{\max }=80 \mathrm{~d}$ $(=2000 \mathrm{~mm})$. The amplitudes corresponding to these wavelengths are given by $y_{\min }=d \tan \alpha=$ $25 \mathrm{~mm} \cdot \tan \alpha$ and $y_{\max }=20 d \tan \alpha=500 \mathrm{~mm} \cdot \tan \alpha$, respectively. In general, the amplitude corresponding to wavelength $L_{n}$ can be written as

$$
y_{n}=\frac{1}{4} L_{n} \tan \alpha=n d \tan \alpha ; \quad L_{n}=4 n d
$$

where $n$ is the number of considered harmonics, with $n=1$ chosen to correspond to the minimum wavelength; $1 \leqslant n \leqslant 20$. Figure 10 shows the relation between the wavelength and the amplitude of the discrete signal.

Next, the relation between the gradient and the original geometry is considered. The extreme value
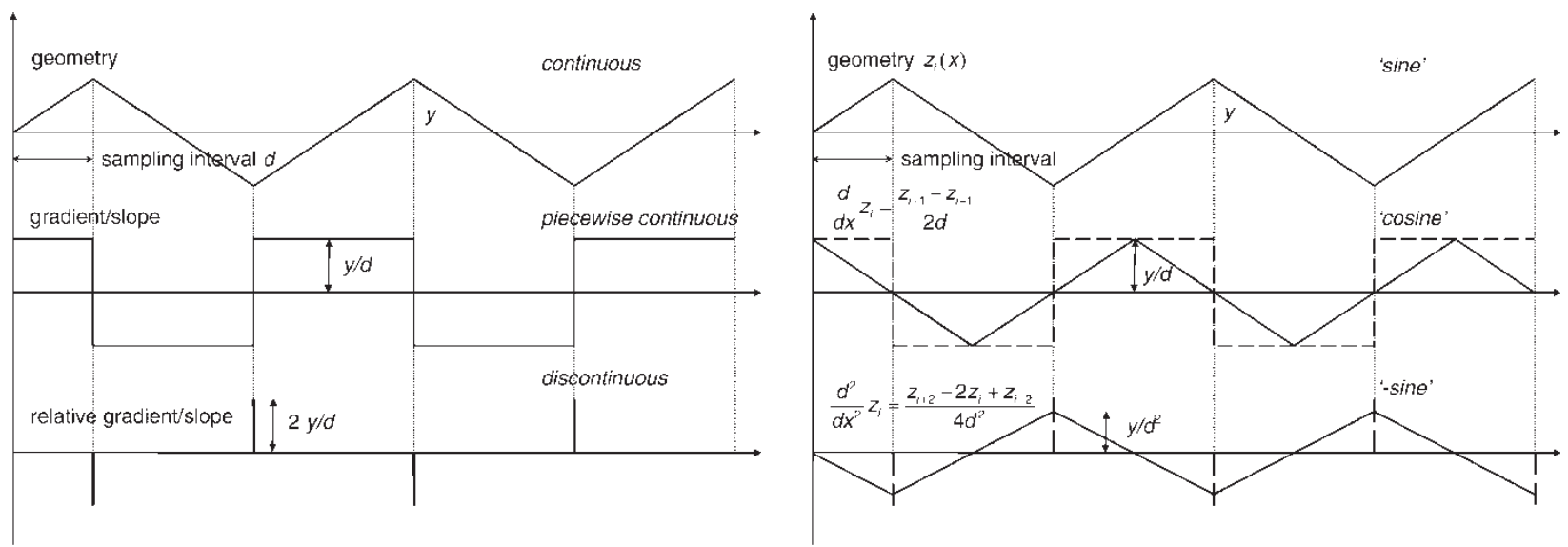

Fig. 9 Discrete harmonic signal with minimum wavelength, discrete gradients and relative discrete gradients (left), and derivatives in the sampling positions (right). 


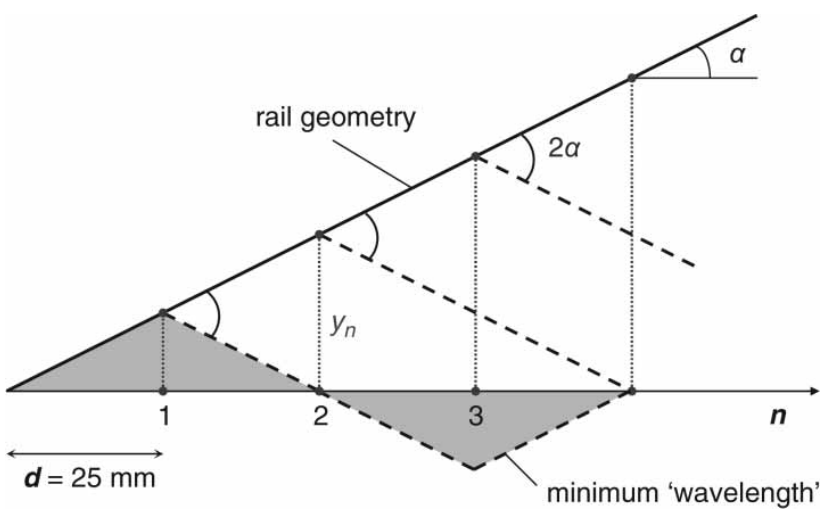

Fig. 10 Geometry development possibilities for a harmonic signal with gradients in each interval at the intervention level

of $z_{i}(x)$ corresponding to a standardized gradient $\tan \alpha$ is reached for a harmonic signal with maximum wavelength $L_{\max }=80 n d(2 \mathrm{~m})$ and equals (Fig. 10)

$$
z_{\max }=\frac{1}{4} L_{\max }\left|\frac{\mathrm{d} z}{\mathrm{~d} x}\right|_{\text {norm }} ; \quad \frac{1}{4} L_{\max }=0.5 \mathrm{~m}
$$

where $|\mathrm{d} z / \mathrm{d} x|_{\text {norm }}=\tan \alpha$. In Table 2 , the values of $z_{\max }$ are given for the intervention levels for the gradient, as given in Table 1 . The values obtained with equation (11) are theoretical maxima corresponding to the norm. For practical relevance, in Table 2, the extreme values are also given assuming a more realistic harmonic waveform (described by 80 sampling points) instead of a quadri-linear waveform with wavelength $2 \mathrm{~m}$. Therefore, the values in the fourth column of Table 2 give an indication of the relation between traditional standards in terms of vertical tolerances and the proposed assessment in terms of discretized gradients.

Finally, the relation between the first and second derivatives of a discrete signal is addressed. In Fig. 11(a), the relation is shown between the discrete gradient and the relative discrete gradient for a discrete signal with limited gradient $\tan \alpha$. A linear and directly proportional relation exists, with a bandwidth $2 \tan \alpha$ (this bandwidth is symmetric or the possible deviation equals the limit value). It may be concluded that for a discrete signal, a limitation of the gradient implies a limitation of the relative gradient at the sampling positions. This is due to the fact that a linear interpolation between the sampling positions is used, thus limiting the transition in angle to the sum of the minimum and maximum angles or twice the allowed angle.

A similar relation exists between the first and second derivatives of a discrete signal, when its first derivative is limited. This is shown in Fig. 11(b). Given a maximum value for the first derivative $y / d$, the limits of the second derivative, in absolute sense (i.e. irrespective of the actual slope), are given by

$$
\begin{aligned}
\frac{\mathrm{d}^{2}}{\mathrm{~d} x^{2}} z_{i} & =\frac{\left(z_{i+2}-z_{i}\right) / 2 d-\left(z_{i}-z_{i-2}\right) / 2 d}{2 d} \\
& = \pm \frac{y / d \pm y / d}{2 d}= \pm \frac{y}{d^{2}}
\end{aligned}
$$

For a standardized maximum slope $\alpha$ and an actual slope $\theta$, the maximum relative slope is given by $\theta \pm \alpha$ (Fig. 11(a)) or the bandwidth is twice the norm. The second derivative has a smaller bandwidth. For a standardized maximum value of the first derivative $y / d$ (corresponding to $\alpha$ ) and an actual value $\mathrm{d} z / \mathrm{d} x$ of the first derivative, the maximum value for the second derivative is given by

$$
\frac{\mathrm{d}^{2}}{\mathrm{~d} x^{2}} z_{i}=\frac{\mathrm{d} z / \mathrm{d} x \pm y / d}{2 d} \quad\left(=\frac{\mathrm{d} z / \mathrm{d} x \pm \tan \alpha}{2 d}\right)
$$

Or, the bandwidth is inversely proportional to the sample interval and directly proportional to the standardized slope. Where a doubling of the relative gradient may occur, a reduction of the second derivative with $1 / d$ is found (Fig. 11(b)).

Table 2 Relation between an assessment method based on gradients and the traditional method based on tolerances

\begin{tabular}{llll}
\hline & $\begin{array}{l}\text { Gradient } \\
\text { intervention }\end{array}$ & $\begin{array}{l}\text { Theoretical tolerance } \\
\text { on } 1 \mathrm{~m} \text { basis }\end{array}$ & $\begin{array}{l}\text { Tolerance on } 1 \mathrm{~m} \\
\text { basis for a 'single-wave }\end{array}$ \\
$\begin{array}{l}\text { Speed interval } \\
(\mathrm{km} / \mathrm{h})\end{array}$ & level $\left|\frac{\mathrm{d} z}{\mathrm{~d} x}\right|_{\text {norm }}(\mathrm{mrad})$ & $z_{\max }=0.5\left|\frac{\mathrm{d} z}{\mathrm{~d} x}\right|_{\text {norm }}(\mathrm{mm})$ & form' $z_{\max } \approx \frac{1}{\pi}\left|\frac{\mathrm{d} z}{\mathrm{~d} x}\right|_{\text {norm }}(\mathrm{mm})$ \\
\hline $0-40$ & 3.20 & 1.60 & 1.02 \\
$40-80$ & 2.40 & 1.20 & 0.76 \\
$80-140$ & 1.80 & 0.90 & 0.57 \\
$140-200$ & 0.90 & 0.45 & 0.29 \\
\hline
\end{tabular}





Fig. 11 Implicit limitation of relative gradient by limitation of the gradient of a discrete signal (left) and implicit limitation of second derivative by limitation of the first derivative of the signal (right)

7 RELATIONSHIP BETWEEN THE

INTERVENTION LEVEL FOR THE

GRADIENT OF THE RAIL GEOMETRY AND

THE LOCAL MAXIMUM DYNAMIC WHEEL-RAIL CONTACT FORCE

Equation (8) described the dynamic wheel-rail contact forces in terms of the second spatial derivative of the discrete signal of the rail geometry. Substitution of equation (13) yields the following expression for the dynamic contact forces in terms of the first spatial derivative of the discrete signal

$$
F_{\text {dyn }}(x)=\beta M_{\text {track }} V^{2} \frac{1}{2 d}\left(\frac{\mathrm{d} z}{\mathrm{~d} x} \pm \tan \alpha\right)
$$

Thus, a linear relationship is found between the dynamic contact force and the first spatial derivative of the weld geometry, however, within a certain bandwidth. The maximum of expression (14) is found (with $|\mathrm{d} z / \mathrm{d} x|_{\text {norm }}=\tan \alpha$ ) by

$$
F_{\text {dyn,max }}=\beta M_{\text {track }} V^{2} \frac{1}{d}\left|\frac{\mathrm{d} z}{\mathrm{~d} x}\right|_{\text {norm }}
$$

An important result has been obtained. Equations (14) and (15) establish a relationship (within a certain bandwidth) between the gradient of the discrete longitudinal contact geometry and the corresponding force between wheel and rail, when the gradient is standardized.
In equation (15), the full bandwidth was used to determine the maximum (in Fig. 11(b), the boundaries of the band were used). From a statistical point of view, it can be argued that the discrete gradients have a distribution which is, over a large part of the gradient domain, close to normal with zero mean (as will be shown in section 9). Therefore, a zero bandwidth will be closest to reality when maximum contact forces for a large number of welds are considered. Using the central relation in Fig. 11(b) with zero bandwidth yields

$$
F_{\text {dyn,max }}=\beta M_{\text {track }} V^{2} \frac{1}{2 d}\left|\frac{\mathrm{d} z}{\mathrm{~d} x}\right|_{\text {norm }}
$$

Equation (15) gives the deterministic maximum contact force corresponding to a specific sampled geometry. The statistical uncertainty introduced by

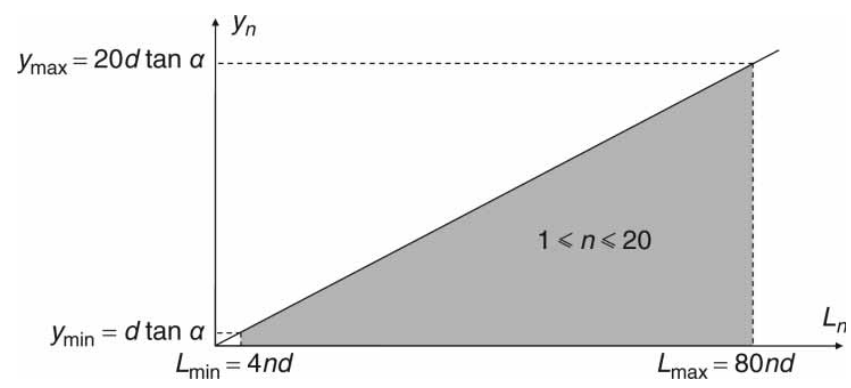

Fig. 12 Linear relation between the wavelength and the amplitude of a quadrilinear harmonic signal 
the bandwidth can be accounted for by validation of a new calibration factor that will be introduced in the following; this way, it makes no difference whether expression (15) or (16) is used.

Now, again the particular case of a longitudinal weld geometry consisting of a purely harmonic signal with a discrete gradient in each sampling interval corresponding to the intervention level is considered (Figs 9 and 10). The maxima of first and second derivatives are then given by (Fig. 9(b))

$$
\frac{y_{n}}{n d}=\tan \alpha
$$

and

$$
\frac{\frac{y_{n}}{n^{2} d^{2}}=\tan \alpha}{n d}
$$

respectively, where the linear relation between amplitude and wavelength (10) was used. Substitution of equation (17) in equation (14) and equation (18) in equation (9) yields

$$
\begin{aligned}
& F_{\text {dyn,max }}=\beta M_{\text {track }} V^{2} \frac{\tan \alpha}{d} \\
& F_{\text {dyn,max }}=\beta M_{\text {track }} V^{2} \frac{\tan \alpha}{n d}
\end{aligned}
$$

A comparison of expressions (19) and (20) shows that the expression for the maximum dynamic force obtained from the second derivative depends on the wavelength (or, alternatively, on $n$ ), whereas the expression obtained by substitution of the relation between first and second derivatives is independent of the wavelength. This result is also valid for a harmonic signal described by more sampling points per wavelength. However, results for a harmonic signal cannot be extended automatically to an arbitrary geometry. The sampled geometry (with finite record length) can be decomposed into a finite number of harmonics using the discrete Fourier transform (DFT). Because the Fourier transform is a linear operation, the total dynamic response can be found as the superposition of the responses to each component. However, a constraint on the gradient of the signal does not imply a constraint on the gradient of each of the components in the summation. Therefore, result (15) is more general.

Equations (15) and (16) may be summarized in a general form, with a dimensionless calibration factor $\gamma$

$$
F_{\mathrm{dyn}, \max }=\gamma M_{\mathrm{track}} V^{2} \frac{1}{d}\left|\frac{\mathrm{d} z}{\mathrm{~d} x}\right|_{\text {norm }}
$$

$\gamma$ is the dimensionless calibration factor (-) (which may vary per interval for the line-section speed), $M_{\text {track }}$ the equivalent track mass $(\mathrm{kg}), V$ the train speed $(\mathrm{m} / \mathrm{s}), d$ the sampling distance in the averaged and filtered rail geometry signal (m), and $|\mathrm{d} z / \mathrm{d} x|_{\text {norm }}$ the standardized gradient of this signal $(-)$.

Thus, a final relation is established between the maximum dynamic wheel-rail contact forces and the standardized gradient of the discrete rail geometry.

\section{VALIDATION OF THE INTERVENTION LEVELS AND EXTENSION TO HIGH-SPEED AND HEAVY-HAUL LINES}

There is an important difference between axle loads for conventional trains and tracks and high-speed and heavy-haul tracks, respectively. For high-speed trains, the static component of the vehicle-track interaction force is much lower $(\sim 170 \mathrm{kN})$, whereas running speeds are much higher $(\sim 300 \mathrm{~km} / \mathrm{h})$ than for conventional tracks (usually $<225 \mathrm{kN}$ with speed $140 \mathrm{~km} / \mathrm{h})$. For heavy-haul tracks, the opposite holds: axle loads are much higher $(\sim 350 \mathrm{kN})$ and running speeds are much lower $(<100 \mathrm{~km} / \mathrm{h})$.

Therefore, allowable dynamic amplifications are much lower for heavy-haul lines than for highspeed lines. However, as the dynamic component of the vehicle-track force (according to equations (9) and (21)) increases quadratically with the speed, weld geometry requirements for high-speed lines need to be much stricter in a consistent approach. Also from a practical point of view, for heavy-haul lines, the rail welds are not a real issue, as rails on these tracks are often ground. The earlier relations among geometrical weld quality, static axle load, and running speed are expressed in the following formula, where $Q$ represents the axle load and equation (21) has been used for its dynamic component

$$
\begin{aligned}
& Q_{\text {tot }}=Q_{\text {stat }}+\gamma \frac{M}{d} V^{2}\left|\frac{\mathrm{d} z}{\mathrm{~d} x}\right|_{\max } \longrightarrow \\
&\left|\frac{\mathrm{d} z}{\mathrm{~d} x}\right|_{\max }<\Delta Q \frac{1}{\gamma} \frac{d}{M} \frac{1}{V^{2}}
\end{aligned}
$$

On the basis of this expression, an intervention level for heavy-haul tracks has been proposed in reference [8]; the focus in this article will be on requirements for high-speed tracks and the validation of intervention levels.

Requirements for high-speed lines can easily be derived on the basis of the geometrical quality of the surface of new rails in longitudinal direction. There is no need for rail welds to have a better geometrical quality than the rails themselves. To determine this quality, geometry measurements have been carried out on new straightened rails; a population of 100 


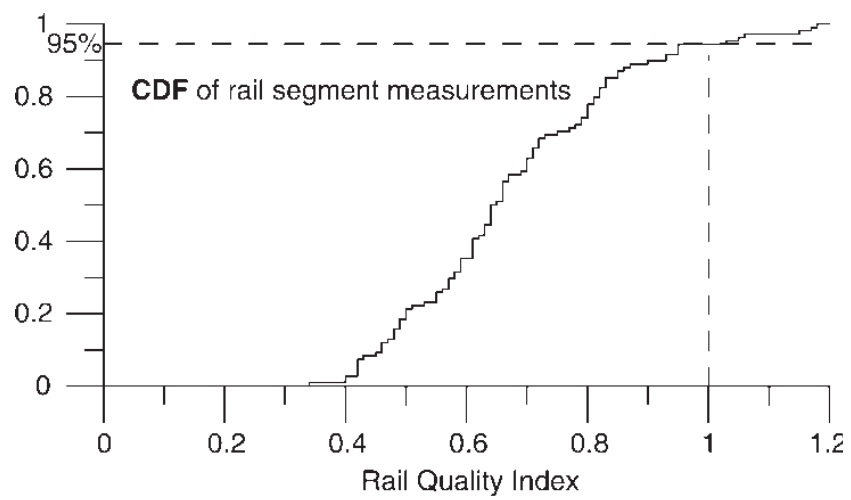

Fig. 13 Cumulative distribution function of the QI (at $300 \mathrm{~km} / \mathrm{h}$ ) for new vertical rail geometry (100 segments of $1 \mathrm{~m}$ )

segments of $1 \mathrm{~m}$ length has been measured [9]. The distribution function of the calculated rail QI for these segments is given in Fig. 13. This rail QI has been calculated assuming an intervention level of $0.7 \mathrm{mrad}$ for the discretized rail gradient.

Assuming this intervention level, 95 per cent of the rail measurements satisfy the requirements and have an index less than 1. Therefore, $0.7 \mathrm{mrad}$ may be taken as an appropriate value for the intervention level of the weld geometry gradient for running speeds up to $300 \mathrm{~km} / \mathrm{h}$ (and higher). In fact, this accuracy is very close to the maximum obtainable accuracy in welding; often, special grinding equipment (e.g. a grinding train) is necessary to realize this accuracy [9]. In Fig. 14, cumulative distributions are shown of rail weld geometry measurements on the Dutch high-speed line after manufacturing and after grinding with a grinding train (one single passage with two types, leading to a QI less than 2 for 99 and 96 per cent of the populations, respectively).

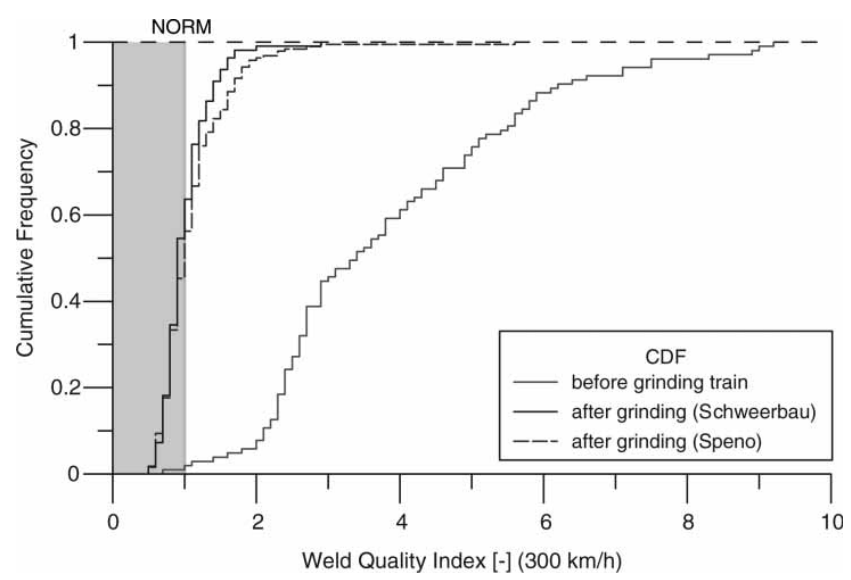

Fig. 14 Influence of grinding with a grinding train on the cumulative distributions of the QI (at $300 \mathrm{~km} / \mathrm{h}$ ) for welds on high-speed track

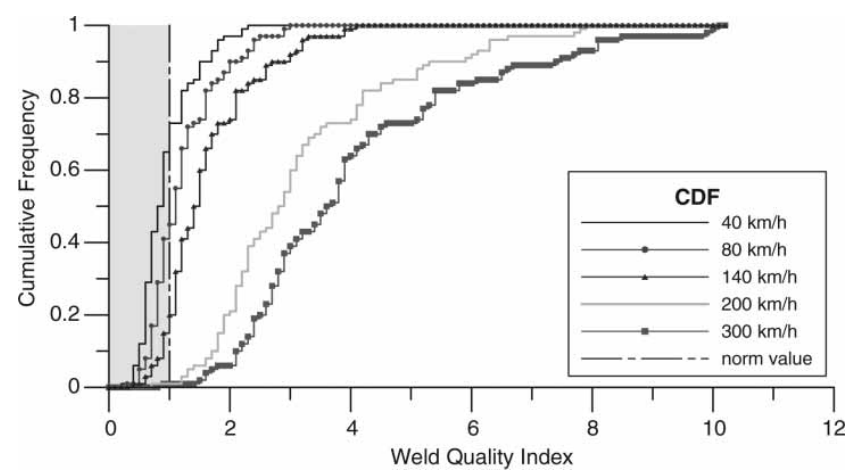

Fig. 15 Cumulative distribution function of the QI for a weld population of 110 welds at different line-section speeds

\section{STATISTICAL ANALYSIS OF WELD AND RAIL MEASUREMENT DATA: PARAMETER DISTRIBUTIONS}

The consequences of the proposed new geometrical assessment of rail welds have been studied by performing some statistical analyses on weld measurement data. A sample has been taken with a set of 110 rail weld measurements (these welds have been manufactured with an assessment based on the principle of tolerances).

In Fig. 15, the cumulative distribution functions for the weld QI are shown for the earlier introduced train speed intervals; also the high-speed norm has been added. The norm value 1 for the QI is also shown. The QI range less than 1 is shaded; parts of the functions situated in this area are 'approved'. Some additional statistical information is given in Table 3. It is observed that the differentiation of standards to the line-section speed has an important influence on the relative percentage of acceptance.

In Fig. 16 at the left-hand side, the QI is shown for the weld population, plotted against the standard normal variable. If the resulting function is a straight line, this implies that the QI has a normal distribution (with mean and standard deviation following from the graph). In Fig. 16 (left), the best linear fit is shown; this is shown for $300 \mathrm{~km} / \mathrm{h}$ only (using the least-squares method), as all graphs are basically derived from the same data set and have the same

Table 3 Statistics for a population of 110 vertical geometry measurements of rail welds

\begin{tabular}{lccccc}
\hline Line speed $(\mathrm{km} / \mathrm{h})$ & 40 & 80 & 140 & 200 & 300 \\
\hline Mean QI & 0.87 & 1.16 & 1.55 & 3.10 & 3.98 \\
Standard deviation & 0.43 & 0.57 & 0.76 & 1.53 & 1.97 \\
Coefficient of variation & 0.49 & 0.49 & 0.49 & 0.49 & 0.49 \\
Acceptance: QI in two digits & $74 \%$ & $46 \%$ & $23 \%$ & $2 \%$ & $2 \%$ \\
QI with one digit & id & $55 \%$ & $33 \%$ & id & id \\
\hline
\end{tabular}



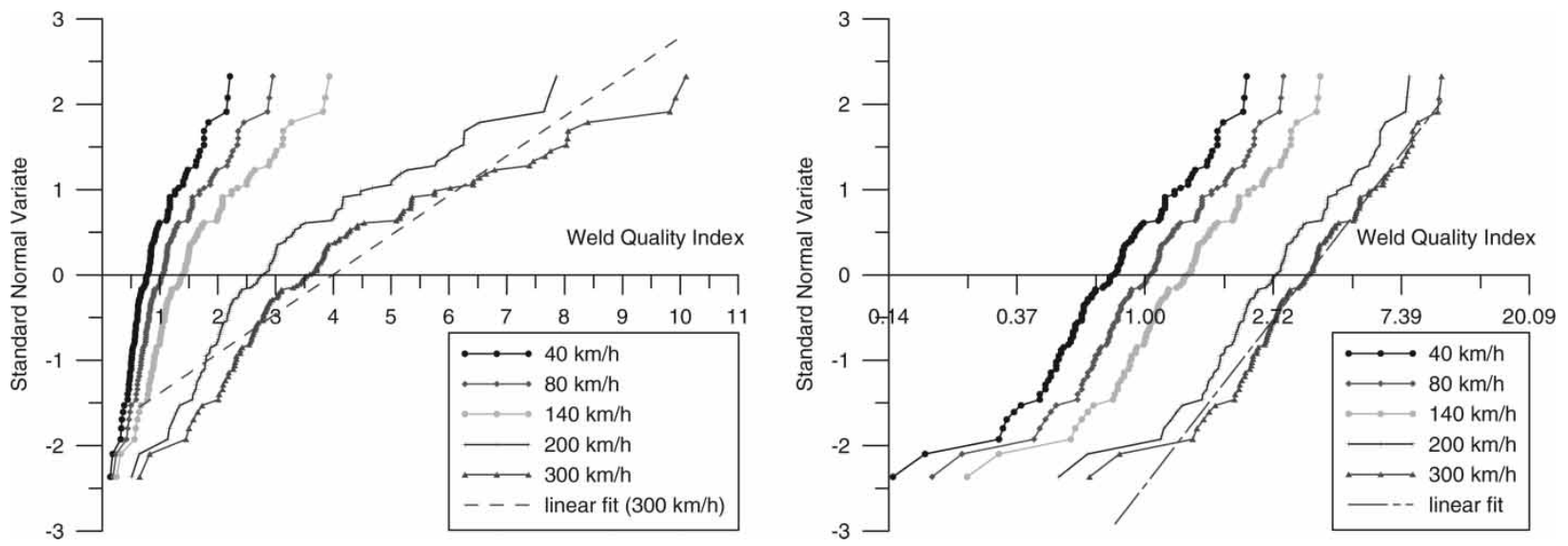

Fig. 16 Standard normal variable versus the weld QI. Linear scale (left) and logarithmic scale (right) for a population of 110 rail welds and different line-section speeds
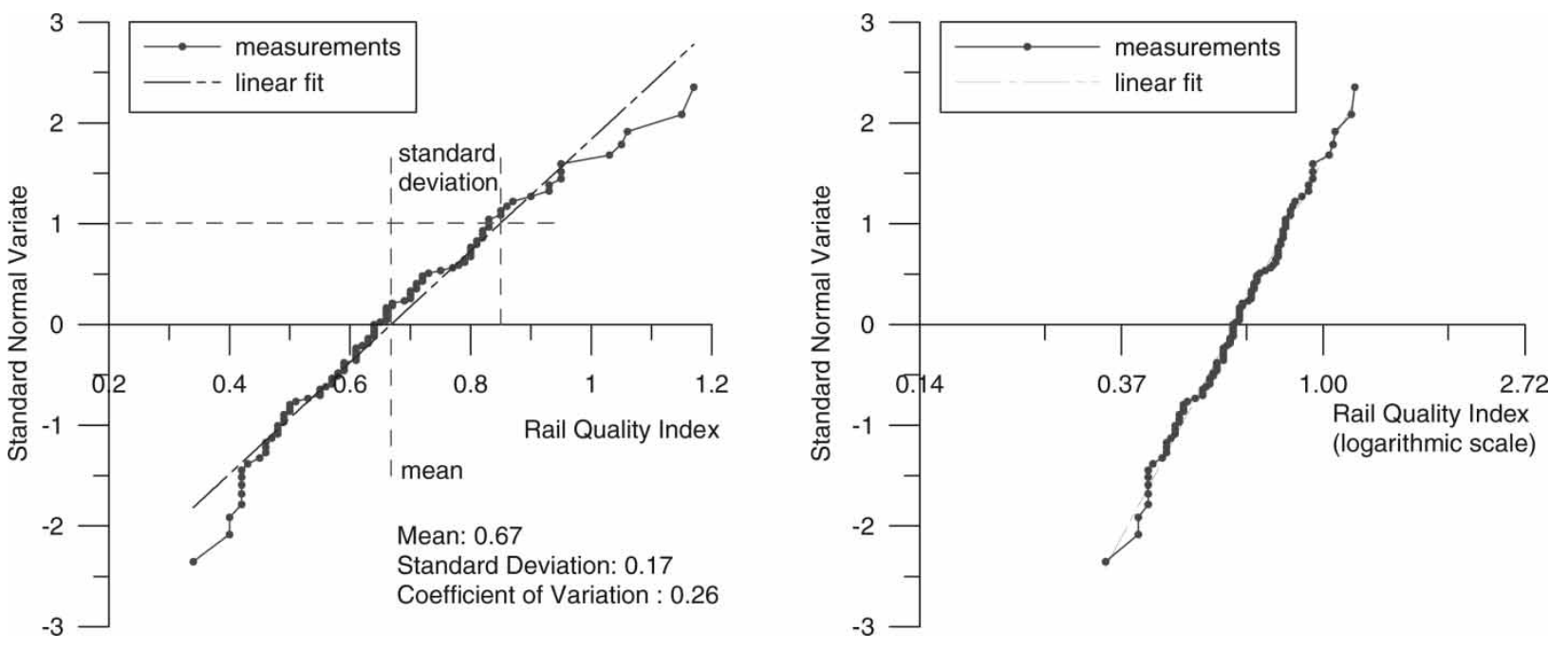

Fig. 17 QI of 100 rail segments versus standard normal variable on linear (left) and logarithmic (right) scales
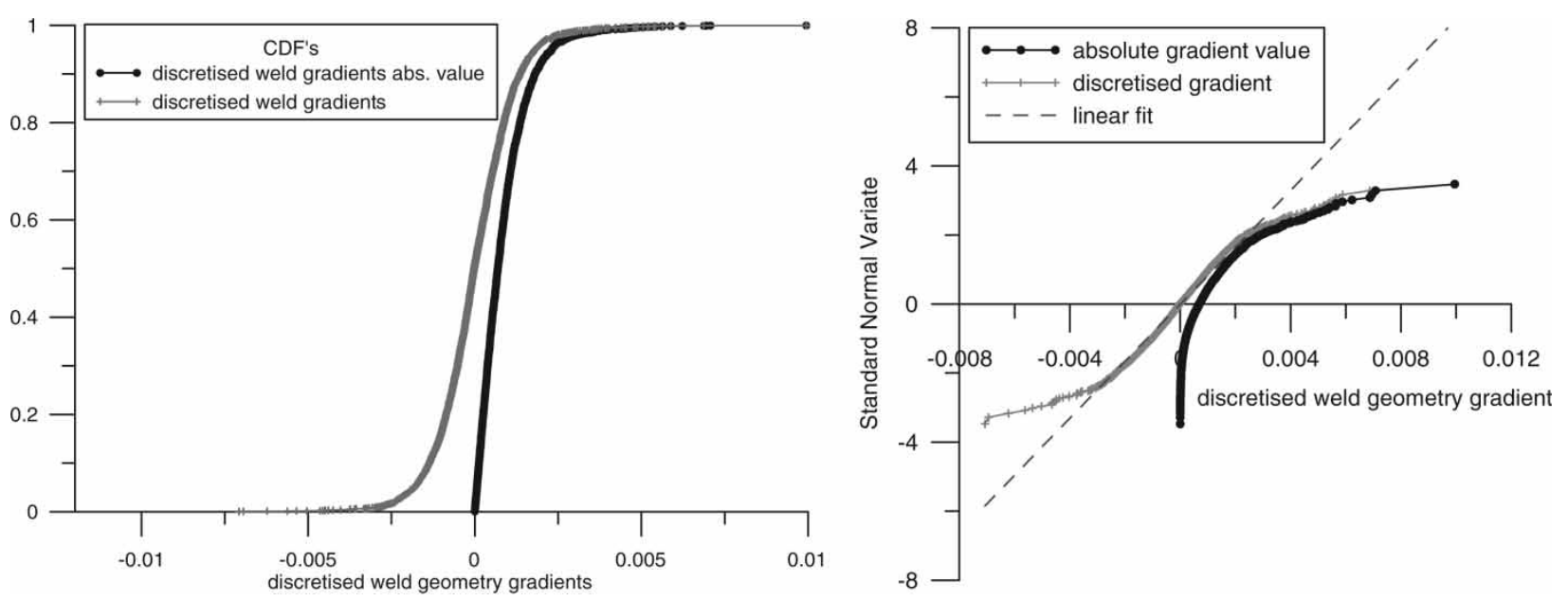

Fig. 18 Left: cumulative distribution functions of discretized weld gradients. Right: discretized weld geometry gradient versus standard normal variable 

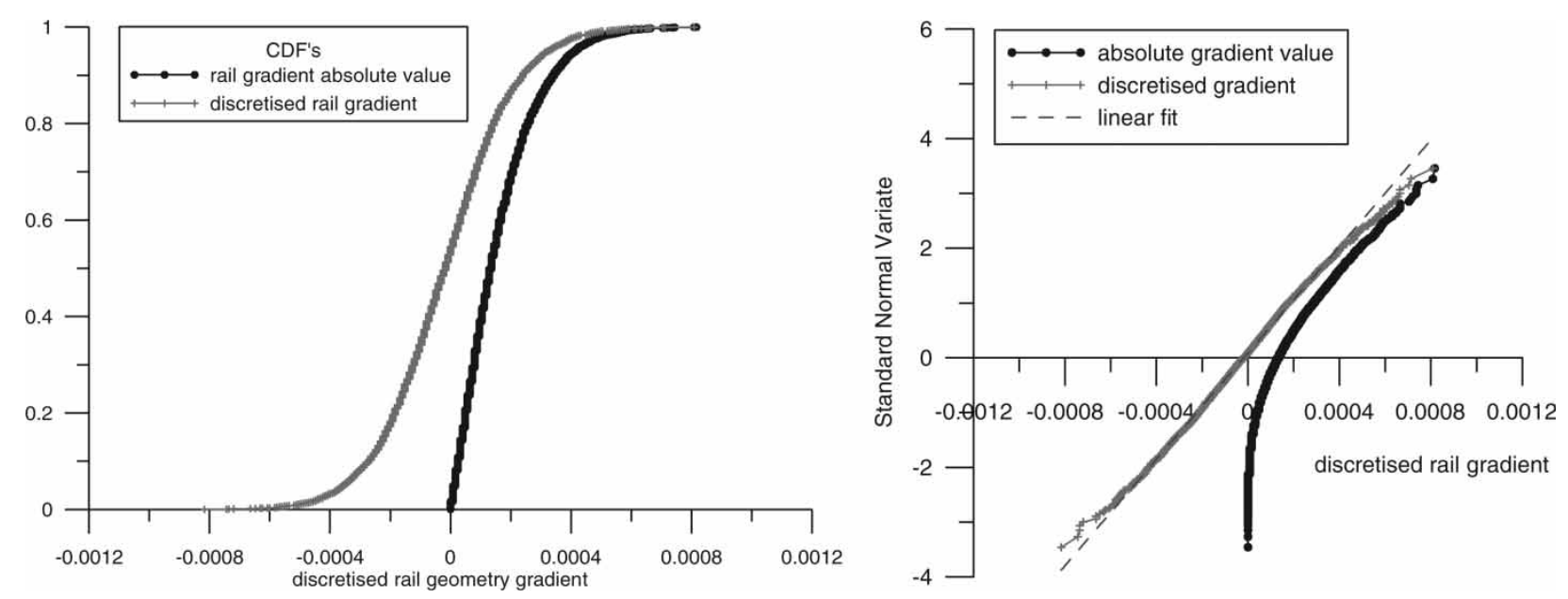

Fig. 19 Left: cumulative distribution functions of discretized rail gradients. Right: discretized rail geometry gradient versus standard normal variable

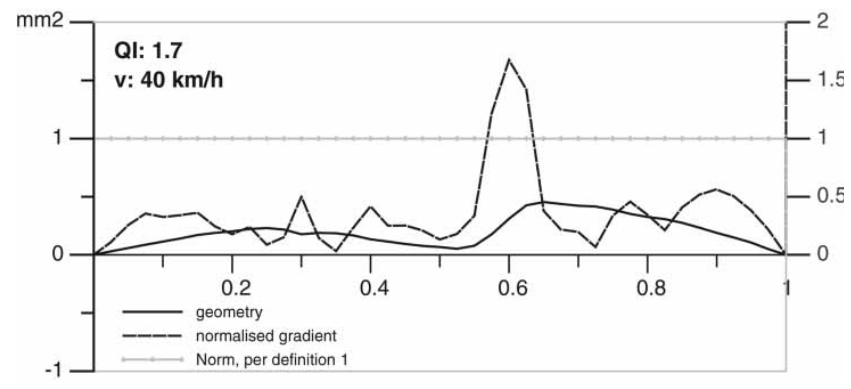

Fig. 20 Possible screen output of a measuring device for the assessment of the geometry of rail welds

'shape'. It is clear that a linear fit is a bad approximation. The same procedure has been repeated for the right-hand plot in Fig. 16 using a logarithmic scale for QI. A linear relation between the standard normal variable and $\ln (\mathrm{QI})$ implies a log-normal distribution of the QI. The result is much closer to a linear relation; neglecting the 'tails' (which contain only very few data points), the approximation by a linear function is rather accurate. Furthermore, in the lower tail, the QIs with a low value, or the almost perfect welds, are found; these are not so much of interest.

Comparison of the left and the right plots in Fig. 16 shows that the distribution of the QI (and thus the maximum slope in the weld geometry) can be approximated as log-normal. This could have been expected, as the QI follows directly from the maximum slope in the weld geometry. When considering the discretized gradient itself as a normal random variable, this maximum should have an extreme value distribution, which always has some exponential form. If the gradient would be exactly normally distributed, its maximum would have a type I - Gumbel distribution. The fact that the absolute values of the gradient are taken and that the gradient itself does not have an exactly normal distribution (which is shown later in this section) disturbs this picture.

In Fig. 17, the same procedure has been repeated, but now for the QI of the rail segments. Clearly,

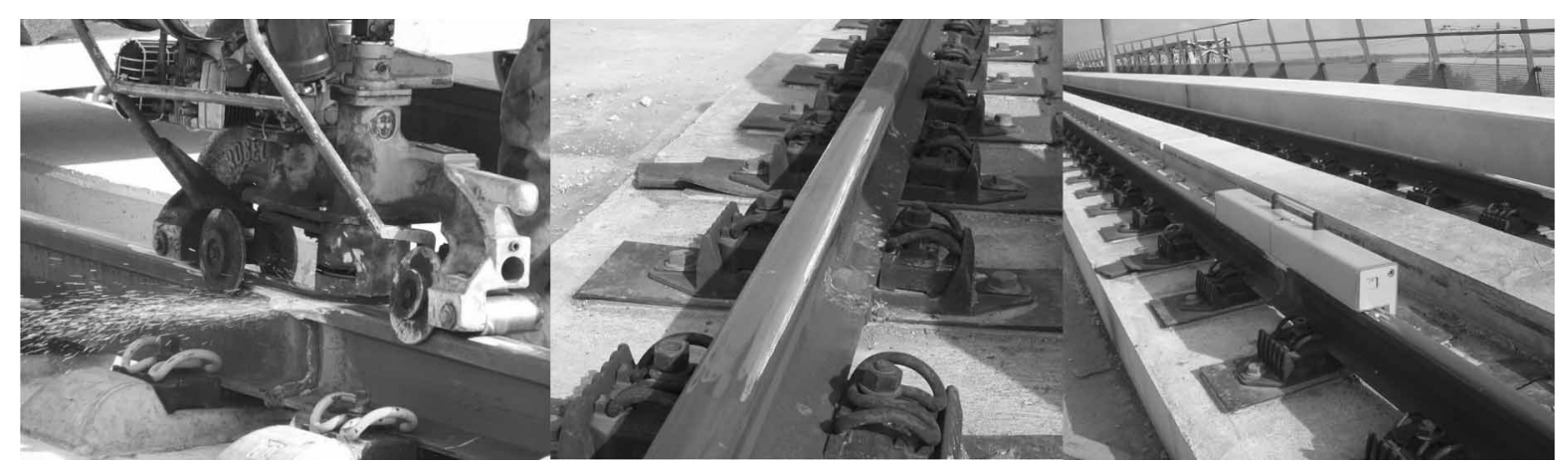

Fig. 21 Semi-mechanical grinding of the rail weld surface in longitudinal direction, ground rail, and electronic geometry measurement 
the log-normal distribution is a rather accurate description for this variable.

In Figs 18 and 19, the behaviour of the discretized weld and rail gradients are analysed (not their respective maxima). Distribution functions are shown in the left plot; in the right plot, their values have been plotted against the standard normal variable. A log-normal distribution is not possible because of the presence of negative gradient values. It can be concluded that for the discretized gradients of the welds, the normal distribution is accurate, with mean value exactly at zero, when the tails (where extreme values occur) are disregarded; in the interval [ $-0.003,0.003 \mathrm{mrad}]$, the normal distribution fits well. This conforms the expectations, as the gradient is a random variable. The limitation of the validity domain of the normal distribution is probably due to an implicit limitation of this random variable introduced by the vertical tolerances, which causes the deviating 'tails'. This is confirmed in Fig. 19; the discretized rail geometry gradient itself has an almost perfect normal distribution with a mean value exactly at zero.

\section{PRACTICAL IMPLEMENTATION OF THE PROPOSED ASSESSMENT METHOD}

In practice, after welding of rails, the quality of the geometry can be measured using an electronic digital straightedge. In its processor, the routines for the calculation of the QI can be programmed. The device then samples the rail geometry and plots the QI on a screen. With the help of this output, the geometry can be optimized such that the standards are met. An example of such a screen output is given in Fig. 20. At the location where the longitudinal rail geometry shows a relatively large angle, the gradient, normalized with the norm value for $40 \mathrm{~km} / \mathrm{h}$, exceeds the norm (the QI is 1.7), and the weld should be ground before acceptance.

In Fig. 21, the steps for the assessment of rail welds are shown in practice.

\section{CONCLUSIONS AND CONSIDERATIONS ON FURTHER RESEARCH}

An assessment method for the geometrical assessment of rail welds has been proposed, based on a limitation of the maximum of the gradient of the discrete measurement signal. The limitation of the gradient implicitly limits the maximum dynamic wheel-rail contact force at the weld. This assessment method has been standardized in the Netherlands (2005).
However, there remain several open questions and aspects, which need further experimental and theoretical research, which will be carried out in the near future. This will allow for the validation of the new standards. The subjects to be investigated are as follows.

1. The level of acceptance, which was chosen empirically on the basis of experience (at least for conventional tracks), should be coupled with life-cycle analyses, where the most critical component(s) in a track system should be determined. In this way, an optimization of the intervention levels can be performed.

2. Measurements should be carried out to verify and validate equation (21), the basic tool for new weld assessment, with respect to the coefficient for obtaining quantitative results for the dynamic wheel-rail interaction force.

3. Monitoring of the initially realized weld geometry as a function of the passed tonnage (improvement versus deterioration of the weld quality as a result of plastic deformations and wear) is an important issue.

4. This holds also for the monitoring of the overall track quality (the other track components such as rail fasteners, sleepers, and ballast) versus initial weld quality as a function of passed tonnage.

\section{REFERENCES}

1 Steenbergen, M. J. M. M. and Esveld, C. Voorstel voor normering van lassen in spoorstaven. Report 7-04220-7, ISSN 0169-9288, Delft University of Technology, Delft, 2004.

2 Steenbergen, M. J. M. M., Esveld, C., and Dollevoet, R. P. B. J. New Dutch assessment of rail welding geometry. Eur. Railway Rev., 2005, 11, 71-79.

3 Anon. Manchester benchmarks for rail vehicle simulation. Veh. Syst. Dyn., 1998, 30, 295-313.

4 De Man, A. P. Dynatrack, a survey of dynamic railway track properties and their quality, 2002 (Delft University Press, Delft, The Netherlands).

5 Jenkins, H. H., Stephenson, J., Clayton, G. A., Morland, G. W., and Lyon, D. The effect of track and vehicle parameters on wheel/rail vertical dynamic forces. Railway Eng. J., January 1974, 2-16.

6 DARTS-NL. Dynamic analysis of a rail track structure, available from www.esveld.com, 2005.

7 Esveld, C. Modern railway track, 2nd edition, 2001, p. 56, 316, 570 (MRT-Productions, Zaltbommel, The Netherlands).

8 Esveld, C. and Steenbergen, M. J. M. M. Force-based assessment of weld geometry. In Proceedings of 8th International Heavy Haul Conference, Rio de Janeiro, Brazil, 14-16 June 2005, pp. 51-58.

9 Esveld, C. Measurements of rail and weld geometry on HSL-South, 2005 (ECS, Zaltbommel, The Netherlands). 